\title{
Quick System Design of Vesicle-Based Active Transport Molecular Communication by Using a Simple Transport Model ${ }^{\text {出 }}$
}

\author{
Nariman Farsad, Andrew W. Eckford ${ }^{\mathrm{a}}$, Satoshi Hiyama and Yuki Moritani ${ }^{\mathrm{b}}$ \\ ${ }^{a}$ Nariman Farsad and Andrew W. Eckford are with the Department of Computer Science \\ and Engineering, York University, 4700 Keele Street, Toronto, Ontario, Canada M3J \\ 1P3.Emails: nariman@cse.yorku.ca, aeckford@yorku.ca \\ ${ }^{b}$ Satoshi Hiyama and Yuki Moritani are with Research Laboratories, NTT DOCOMO \\ Inc., Yokosuka, Kanagawa, Japan. Emails: hiyama@nttdocomo.co.jp, \\ moritani@nttdocomo.co.jp
}

\begin{abstract}
This paper will provide a guidepost to design an optimal molecular communication setup and protocol. A barrier to the design of vesicle-based molecular communication nanonetworks is the computational complexity of simulating them. In this paper, a computationally efficient transport model is presented, which could be employed to design active transport molecular communication systems, particularly to optimize the shape of the transmission zone. Furthermore, a vesicular encapsulation model is presented as an addition to the transport model, and it is shown that there exists an optimal vesicle size for each molecular communication channel. As an application, our transport model is used to estimate the channel capacity of a molecular communication
\end{abstract}

\footnotetext{
This paper was published in Nano Communication Networks, doi:10.1016/j.nancom.2011.07.003, 2011. In case of discrepancies, the version in the journal is correct. Material in this paper was presented in part at the IEEE INFOCOM 2011 Workshops: International Workshop on Molecular and Nano-Scale Communications (MoNaCom), Shanghai, China, 2011
} 
nanonetwork in a computationally efficient manner compared to traditional Monte Carlo techniques. Moreover, it is shown that the derived optimal vesicle size maximizes channel capacity.

Keywords: Molecular communication, information theory, system design

\section{Introduction}

Inspired by living organisms, molecular communication [1] is one approach to communication at small scales. In molecular communication, as in intercellular and intracellular communication, molecules are used to transfer information over very small distances, typically on the order of micro- or nanometers. The information can be encoded into the type of the molecule [2], the number or concentration of molecules $[3,4,5]$, or the time of release $[6,3]$. Information carrying molecules can also be encapsulated inside lipid vesicles or liposomes. Vesicular encapsulation compartmentalizes information molecules from the propagation environment and provides a generic architecture to transport diverse types of information molecules, independent of their biochemical and physical characteristics [1]. It also protects information molecules from denaturation (e.g., molecular deformation and cleavage caused by enzymatic attacks or changes in $\mathrm{pH}$ of the outer aqueous phase) in the propagation environment [1]. In [7] a chemical assembly line for generation of lipid vesicles and encapsulation process is presented.

The released molecules or lipid vesicles encapsulating the molecules can propagate from the transmitter or the source to a receiver or the destination using one of the two major propagation schemes: passive transport and active transport $[8,5]$. In passive transport, the information carrying particles 
diffuse in the confined microfluidic channel and follow a Brownian motion from the transmission zone to the receiver zone. In active transport, the information particles are transported actively using molecular motors such as kinesin. For example in [9], microtubule filaments moving over a glass substrate covered with stationary kinesin motors is proposed as an active transport scheme for molecular communication systems. In this work we consider only the active transport propagation scheme proposed in $[9,10]$.

Because of the complexity of molecular communication systems as well as the gaps in the underlying theoretical and mathematical frameworks, researchers sometimes rely on computer simulations to study systems. For example, Monte Carlo simulations are employed in $[8,5]$ to study achievable information rates. However, running simulations can be time consuming and computationally intensive. Therefore, it is desirable to find a computationally efficient mathematical framework for studying these systems. Deriving a mathematical model, specially for molecular communication in confined space, is extremely difficult because of the complexity and dependencies present in these systems.

Notable works employing simulation or mathematical models to study molecular communication systems include a general formulation of molecular communication as a timing channel under Brownian motion [6, 11], an analysis of information transfer rates using molecular motors [12, 3], mathematical channel models for continuous diffusion [13], binary concentration-encoded molecular communication [4], and a simple model comparing the achievable information rates of passive transport using Brownian motion to that of active transport using microtubule filaments moving over a molecular motor 
track $[8,5]$.

In this work, with the help of some independence assumptions, we first present a simple mathematical representation of active transport molecular communication system in confined space, that can be used to optimize and study these systems. We focus on active transport since in [5] it was shown that over long distances it achieves higher information rate compared to Brownian motion. We then use this model to optimize the shape of the transmission area and study the effects of vesicular encapsulation on achievable information rates. Our contributions in this work are as follows.

- We derive an approximate, but low-complexity, transport model which can be used to study and optimize active transport molecular communication.

- As an application, we use this model to find and verify an optimal design of the loading area; this optimization leads to large improvements in performance, and would be computationally intensive to find without our model.

- We also use our model to study the effects of vesicular encapsulation of information molecules. Based on our transport model, we show that there is an optimal vesicle size for a given communication channel.

- Finally, as another application, we show that our proposed transport model could be used to estimate channel capacity of active transport molecular communication channel in a computationally efficiently manner. 


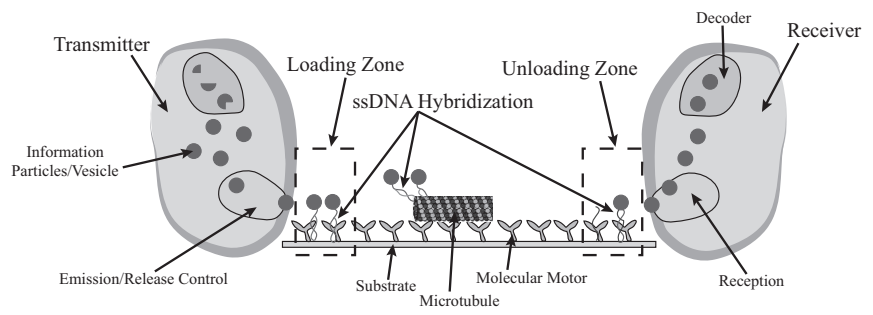

Figure 1: Depiction of the communication environment.

The rest of this paper is organized as follows. In Section 2 we first present a brief overview of the molecular communication environment. We then present our transport model in Section 3. In Section 3.1 we optimize the transmission area using the proposed transport model, while in Sections 3.2 and 3.3 we consider vesicular encapsulation modelling and optimization. As an application, we use our transport model to estimate the channel capacity of an active transport molecular communication system in Section 4, and we show that this method is more computationally efficient than traditional Monte Carlo techniques. We present our concluding remarks in Section 5.

\section{Molecular Communication Environment}

Our molecular communication environment is similar to the ones in $[5$, $8,9]$. We use a rectangular propagation environment (with slightly rounded corners to assist the simulation), consisting of a transmission or loading zone and a receiver or unloading zone. Message-bearing particles originate at the transmission zone, and propagate until they arrive at the receiver zone. Note that message-bearing particles could also be encapsulated inside lipid vesicles by the transmitter before they are released for propagation. In this 
case, the vesicles propagate from the transmitter to the receiver. Information particles or vesicles are initially assumed to be anchored to the transmission zone until microtubule filaments, moving over molecular motor tracks that cover the whole environment, pick up and transport the information carrying particles or vesicles from the transmission zone to the receiver zone.

As in [9], we assume that the microchannel environment is lined with static kinesin molecular motors, and that these motors cause microtubule filaments to propagate along their surface. The motion of the microtubule is largely regular (compared to pure Brownian motion), although the effects of Brownian motion cause random fluctuations in the direction of the motion. The loading and unloading mechanics are assumed to be the same as those proposed in [9]. The particles are anchored to the transmission zone through a single stranded DNA (ssDNA) hybridization bonds, and do not move until they are picked up by a microtubule filament. The pick-up and drop-off mechanisms are also assumed to be carried out through ssDNA hybridization bonds. This process is summarized in Figure 1, and the reader is referred to [9] for detailed explanation.

\subsection{Simulating Active Transport}

Although it has been demonstrated that it is possible to generate and study this molecular communication system in wet labs $[9,10]$, it is very difficult to study these systems from a communication perspective using laboratory experiments. Therefore, previous works have relied on computer simulations to study these systems. In $[14,15]$ the motion of microtubule filaments over stationary kinesin molecular motors was simulated and in $[8,5]$ a complete simulation of the communication system was presented. In this 
work, for our simulation based results, we use the same simulation techniques proposed in [5].

Since the microtubules move only in the $x-y$ directions, and do not move in the $z$ direction (along the height of the channel), we consider a twodimensional simulation of microtubules for $\Delta t$ time intervals. Given some initial position $\left(x_{0}, y_{0}\right)$ at time $t=0$, for any integer $k>0$, the motion of the microtubule is given by the sequence of coordinates $\left(x_{i}, y_{i}\right)$ for $i=1,2, \ldots, k$. Each coordinate $\left(x_{i}, y_{i}\right)$ represents the position of the microtubule's head at the end of the time $t=i \Delta t$, where

$$
\begin{aligned}
x_{i} & =x_{i-1}+\Delta r \cos \theta_{i}, \\
y_{i} & =y_{i-1}+\Delta r \sin \theta_{i} .
\end{aligned}
$$

In this case, the step size $\Delta r$ at each step is an independent and identically distributed (iid) Gaussian random variable with mean and variance

$$
\begin{aligned}
E[\Delta r] & =v_{\mathrm{avg}} \Delta t \\
\operatorname{Var}[\Delta r] & =2 D \Delta t
\end{aligned}
$$

where $v_{\text {avg }}$ is the average velocity of the microtubule, and $D$ is the microtubule's diffusion coefficient. The angle $\theta_{i}$ is no longer independent from step to step: instead, for some step-to-step angular change $\Delta \theta$, we have that

$$
\theta_{i}=\Delta \theta+\theta_{i-1}
$$

Now, for each step, $\Delta \theta$ is an iid Gaussian-distributed random variable with mean and variance

$$
\begin{aligned}
E[\Delta \theta] & =0, \\
\operatorname{Var}[\Delta \theta] & =\frac{v_{\mathrm{avg}} \Delta t}{L_{p}}
\end{aligned}
$$


where $L_{p}$ is the persistence length of the microtubule's trajectory. In [14], these values were given as $v_{\text {avg }}=0.85 \mu \mathrm{m} / \mathrm{s}, D=2.0 \cdot 10^{-3} \mu \mathrm{m}^{2} / \mathrm{s}$, and $L_{p}=111 \mu \mathrm{m}$. Following [14], in case of a collision with a boundary, we assume that the microtubule does not reflect off the boundary, as in an elastic collision, but instead sets $\theta_{i}$ so as to follow the boundary.

The starting location of the microtubule is assumed to be random and uniformly distributed across the entire propagation area. Moreover, the initial directional angle $\theta_{0}$ is selected uniformly at random from the range $[0,2 \pi]$, and microtubules are assumed to be initially unloaded (without any cargoes). We also assume, as proposed in [9], ssDNA hybridization bond is used to anchor the information particles to the transmission area. Similarly, ssDNAs on the surface of the microtubules hybridize with the ssDNAs on the surface of the information particles when a microtubule passes in close proximity, thereby loading the particles onto the microtubule. From experimental observations it is evident that microtubules can load multiple information particles [9]. Therefore, the loading of a particle happens only if a microtubule with available cargo spots passes in close proximity of a particle.

Furthermore, to capture the loading process, in the simulations and the transport model, we use the grid loading structure proposed in [5]. For loading an information particle, the microtubule filament must drive close to the anchored particle. Therefore, we divide the transmission zone into a square grid, where the length of each square in the grid is the same as the diameter of the particles. We then distribute particles randomly and uniformly between the squares in the grid. If a microtubule enters a square which is occupied by a particle, we assume the microtubule loads that particle 


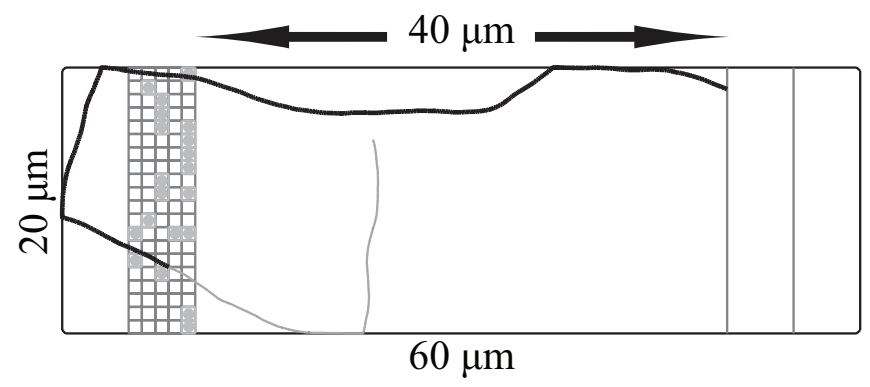

Figure 2: A sample trajectory of active transport. The path of the microtubule is the line in the middle of the rectangular channel. The thinner gray portion corresponds to the path of the unloaded microtubule, while the thicker black portion corresponds to the path of the loaded microtubule. The grid loading structure is on the left hand side and the receiver strip is on the right hand side. The filled squares in the transmission zone represent areas that contain an anchored particle, and empty squares represent areas with no particles. There are a total of 100 squares (i.e. maximum number of particles that could be anchored is 100), 20 of which have anchored particles.

given it has an empty loading slot available. In general, based on laboratory experiments we assume that the microtubules can load multiple particles and the maximum number of particles a microtubule can load is given by half of its length divided by the diameter of the particles. For unloading, we assume all the loaded particles are unloaded as soon as a microtubule enters the receiver zone. Figure 2 shows a sample trajectory with the loading and unloading mechanism. Notice that the transmission and the receiver zone in this figure are rectangular strips across the width of the channel. We refer to this particular shape of the transmission and the receiver zone as the strip transmission zone and the strip receiver zone, respectively. 


\section{Computationally Efficient Transport Model}

In this section we present a computationally efficient transport model for active transport molecular communication. Using our model we find the probability that $Y$ information particles arrive at the destination after $T$ seconds, given $X$ information particles were released by the source.

Assume that our grid transmission zone contains $n$ squares (i.e. the maximum number of particles that can be anchored to the transmission zone is $n$ ). Let $X \leq n$ be the number of particles at the transmission zone in the beginning, and let $Y \leq X$ be the number of particles delivered to the receiver zone after time duration $T$. Let $X_{i}$ be a Bernoulli random variable representing the event where a particle is placed in the $i$ th square for $i=1,2, \cdots, n$. Therefore, if we assume that $X_{i}$ are independent of each other, the probability that an information particle is placed in the $i$ th square is given by

$$
p\left(X_{i}=1\right)=\frac{X}{n}
$$

where particles are distributed uniformly among squares. Note that the independence assumption here is an approximation because it does not satisfy the constraint

$$
X=\sum_{i=1}^{n} X_{i} .
$$

To satisfy this constraint, the dependence of the $X_{i}$ and $X_{i+1}$ must be considered which would make the model complicated.

Let $V_{i}$ be a Bernoulli random variable representing the event that the $i$ th square is visited by the microtubule in a single trip from the receiver zone, to the transmission zone, and back. Therefore, $p\left(V_{i}=1\right)$ represents the 


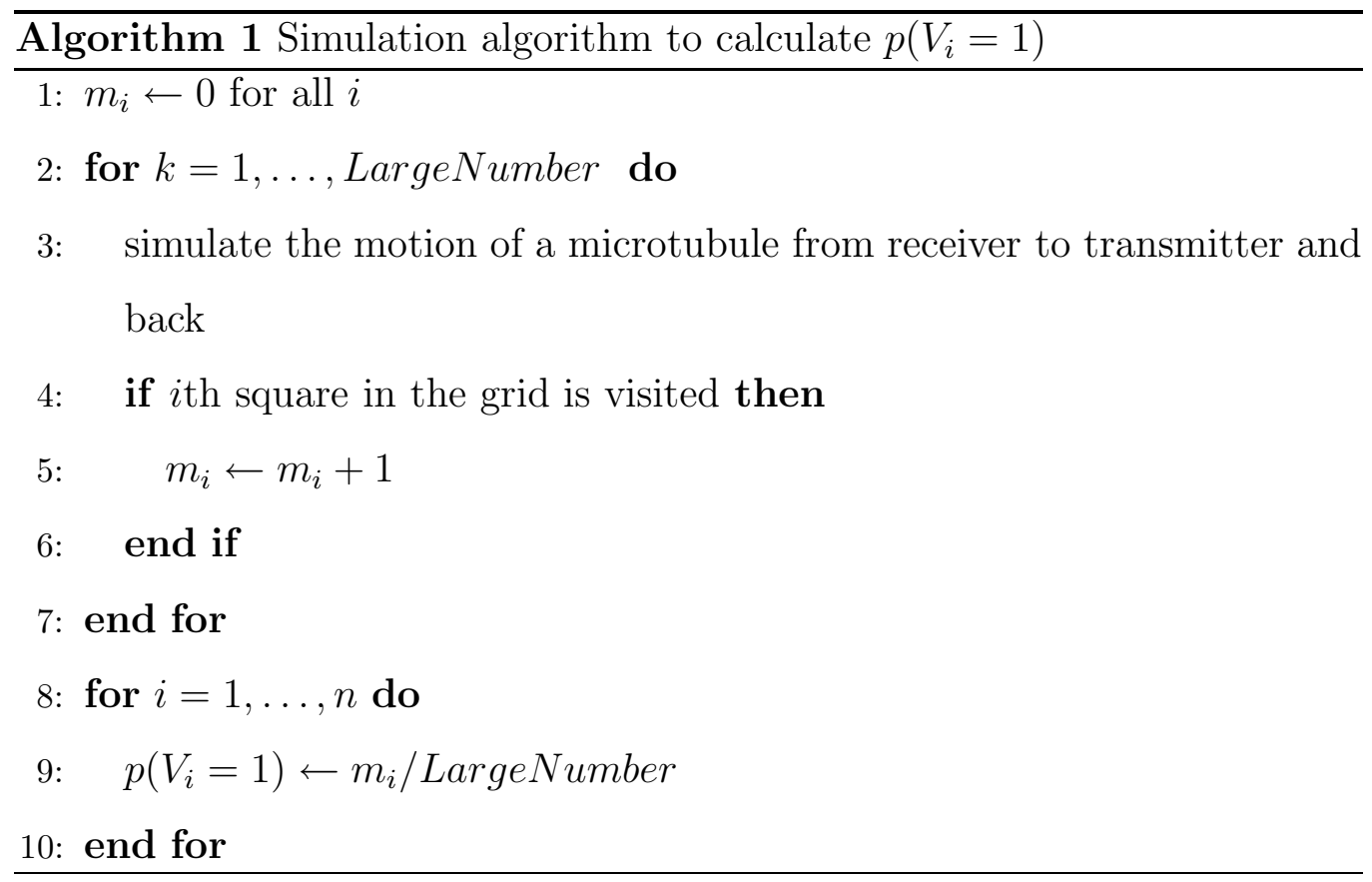

probability that the $i$ th square is visited and $p\left(V_{i}=0\right)$ the probability that it is not visited. This probability distribution can quickly be calculated using simple Monte Carlo simulations for any molecular communication channel as shown in Algorithm 1. For example, the top part of the Figure 3 shows this probability distribution for squares of size $1 \mu \mathrm{m}$ covering the left side of the microchannel. From the probability distribution, it can be seen that the squares close to the walls are visited the most, which is a property of the motion of the microtubules. As shown in $[14,15]$, when the microtubules collide with the walls of the microchannel they follow the boundary with the probability 0.5 .

Let $K$ be another random variable representing the number of microtubule trips between the transmission and the receiver zone in time duration $T$. The probability distribution for $K$ is given in [8], and can be quickly cal- 
culated for any molecular communication channel using simple simulations. Let $V_{i}^{(k)}$ be a Bernoulli random variable representing the event that the $i$ th square is visited at least once by the microtubule during $k$ trips. Therefore,

$$
p\left(V_{i}^{(k)}=1\right)=1-\left(1-p\left(V_{i}=1\right)\right)^{k},
$$

represents the corresponding probability distribution. Here, we have assumed that the probability of visiting a square in any trip is independent and identically distributed random variable $V_{i}$.

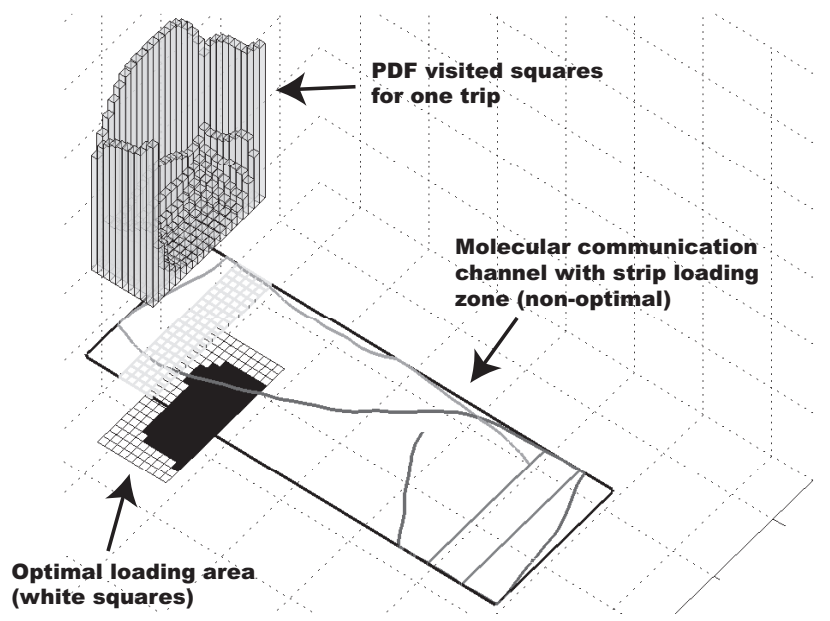

Figure 3: (Top): Probability distribution of $p\left(V_{i}=1\right)$ for squares of size $1 \mu \mathrm{m}$ to the left side of the loading area. (Middle): Strip loading area for $n=100$ squares. (Bottom): Projection of the probability distribution $p\left(V_{i}=1\right)$ on top. The top 100 values of $p\left(V_{i}=1\right)$ are shown in as white squares and they represent the optimal loading area.

Let $D_{i}^{(k)}$ be a Bernoulli random variable representing the event that a particle from the $i$ th square is delivered to the destination after $k$ trips. Then, the probability distribution of $D_{i}^{(k)}$ is given by

$$
p\left(D_{i}^{(k)}=1\right)=p\left(V_{i}^{(k)}=1\right) p\left(X_{i}=1\right)
$$


assuming $p\left(V_{i}^{(k)}=1\right)$ and $p\left(X_{i}=1\right)$ are independent. This independence assumption is not accurate since $p\left(X_{i}=1\right)$ changes depending on the number of particles already delivered in previous trips. In general this assumption becomes less accurate as the number of trips $k$ increases or in other words the channel time duration $T$ increases. Let $Y^{(k)}$ be the total number of particles delivered to the receiver zone during $k$ tips. Then, $Y^{(k)}$ is given by

$$
Y^{(k)}=\min \left(\sum_{i=1}^{n} D_{i}^{(k)}, X\right),
$$

for any given $X$. Since $\sum_{i=1}^{n} D_{i}^{(k)}$ represents a Poisson-Binomial distribution, its corresponding probability distribution can be calculated using [16]. Finally, we can calculate $\operatorname{PMF} f_{Y \mid X}(Y \mid X)$ as

$$
f_{Y \mid X}(Y \mid X)=\sum_{k \in K} p\left(Y^{(k)} \mid X\right) p(k)
$$

where $p\left(Y^{(k)} \mid X\right)$ is the probability mass function of $Y^{(k)}$ given in Equation (12), and $p(k)$ is the probability mass function of $K$, the number of trips between the transmitter and receiver during the time duration $T$.

In the next subsections, we use this transport model to optimize the molecular communication system.

\subsection{Optimizing the Transmission Zone}

We use our transport model presented in previous section, and the fact that the microtubules mostly move along the walls of the molecular communication channel, to optimize the loading area. Recall from top part of the Figure 3 that microtubules mostly move along the walls, and therefore $p\left(V_{i}=1\right)$ (probability that a square is visited in one microtubule trip) is 
higher for squares close to the walls of the molecular communication channel. An information particle is picked up from the transmission zone, and delivered to the receiver zone, if the corresponding square is visited. Therefore, we want to find squares with maximum $p\left(V_{i}=1\right)$, which are squares that have the highest probability of being visited during one trip. In Figure 3 , we plot the probability distribution of all the squares of length $1 \mu \mathrm{m}$ to the left of transmission area (the bar plot on the top). The middle plot in Figure 3 shows our original strip loading area with 100 squares presented in Figure 2. Notice that the squares in the middle of the strip transmission zone have low probability of being visited in a single microtubule trip. The bottom plot shows the projection of the probability distribution function of $p\left(V_{i}=1\right)$. The first 100 squares with highest probabilities are shown as white squares, and the rest of the squares are shown in black. Note that in this projection, the minimum distance between the transmission area and the receiver area is still $40 \mu \mathrm{m}$ and we have not moved the transmission and receiver physically closer. Finally, according to our transport model, this white area is the optimal transmission zone that will give us the highest information rate because probability of visiting and picking up particles is highest.

\subsection{Vesicular Encapsulation}

As mentioned in the introduction, information-carrying particles could be encapsulated inside lipid vesicles to keep them isolated from the propagation environment, thereby preventing destructive chemical reactions that could result in unsuccessful detection at the receiver. However, the effect of vesicular encapsulation on information rate is not clear. For example, is it better to encapsulate all the information molecules inside one giant vesicle 
or inside several smaller ones? Is there an optimal vesicle size for a given molecular communication channel? In this section we present a model for vesicular encapsulation and use the presented transport model in the next section to answer these questions.

We assume egg phosphatidylcholine vesicles (a type of liposome) are used for encapsulation. Furthermore, we assume that lipid vesicles are perfect spheres and the diameter of all the vesicles are exactly the same. Moreover, we assume the number of information molecules per vesicle is deterministic and exact. We make these assumptions to facilitate our analysis, and although these assumptions are strict, relaxing them will not effect the results.

Let $R$ be the inner radius of the lipid vesicle. Then the number of lipid molecules required to create such a vesicle is given by [17]:

$$
m_{R}=\frac{4}{3} \pi N\left(\left(R+3.7 \times 10^{-7}\right)^{3}-R^{3}\right) /(768 \bar{v}),
$$

where $m_{R}$ is the number of lipid molecules required to create a vesicle with radius $R, N$ is the Avogadro's number $\left(6.022 \times 10^{23}\right), R$ is the inner radius of the vesicle in $\mathrm{cm}$, and $\bar{v}$ is the vesicle partial specific volume $(0.9848 \mathrm{~mL} / \mathrm{g})$. For example, using this equation $3.72 \times 10^{7}$ molecules are required to create a single vesicle with diameter of $2 \mu \mathrm{m}$.

Let $M$ be the total number of lipid molecules available at the transmitter for creation of lipid vesicles. Then the total number of lipid vesicles of size $R$ that can be created by the transmitter is given by

$$
n_{R}=\frac{M}{m_{R}} .
$$

Let $x$ be the number of information particles to be sent by the transmitter. Then assuming there are $n_{R}$ vesicles available for encapsulation, there will be 

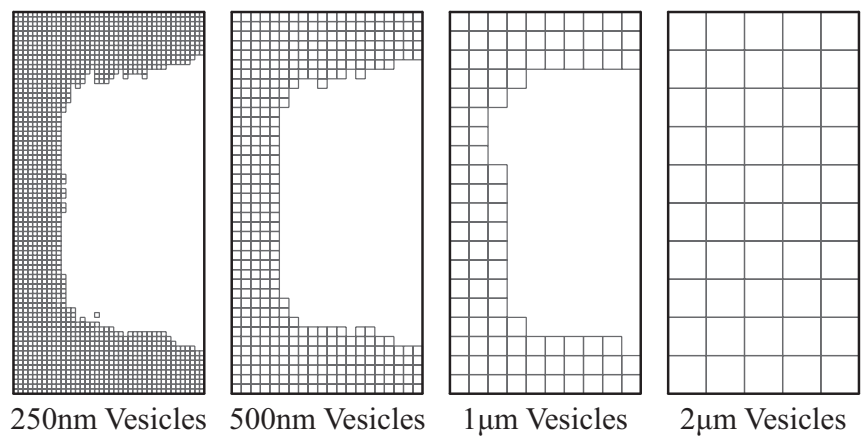

Figure 4: The shape of the optimal transmission area of different vesicle diameters. Each of the four rectangles is the total area that could be dedicated as the transmission area. The dimension of this area is $20 \mu \mathrm{m}$ by $10 \mu \mathrm{m}$. The squares inside each transmission area represent the optimal grid for each vesicle size.

$\left(n_{R}-x \bmod n_{R}\right)$ vesicles with $\left\lfloor x / n_{R}\right\rfloor$ information molecules and $\left(x \bmod n_{R}\right)$ vesicles with $\left\lceil x / n_{R}\right\rceil$ information particles, where (mod) is the modulo operator (i.e. the remainder of the integer division), $\lfloor$.$\rfloor the floor function and \lceil$. the ceiling function. For example, if there are $x=18$ information particles and $n_{R}=5$ vesicles, after the encapsulation process, 2 vesicles encapsulate 3 information particles and 3 vesicles encapsulate 4 information particles.

\subsection{Vesicular Encapsulation Analysis and Optimization}

In this section we use the transport and the vesicular encapsulation models presented in the previous subsection to analyze and optimize vesicular encapsulation. We consider four different diameter values for vesicles: 250nm, $500 \mathrm{~nm}, 1 \mu \mathrm{m}$, and $2 \mu \mathrm{m}$. Our goal is to find the optimal vesicle size. We use the optimization scheme presented in Section 3.1 to optimize the shape of the grid transmission area for each vesicle size. Figure 4 shows the shape of the optimal transmission area for each diameter size. Notice that for the 
$2 \mu \mathrm{m}$ vesicle size, since the square grid occupies the whole area, it is not really optimized.

To make the comparison we rely on Monte Carlo simulation of active transport propagation presented in Section 2.1, and the following parameters: simulation time steps $\Delta T=0.1$ seconds, microtubule diffusion coefficient $D=2.0 \cdot 10^{-3} \mu \mathrm{m}^{2} / \mathrm{s}$, average speed of the microtubule $v_{\mathrm{avg}}=0.5 \mu \mathrm{m} / \mathrm{s}$, and persistence length of the microtubules trajectory $L_{p}=111 \mu \mathrm{m}$. These parameters are all selected based on experimental observations of ssDNA covered microtubules moving over a kinesin covered substrate. Furthermore, for all the subsections that follow, we consider a rectangular propagation environment, with the dimensions $20 \mu \mathrm{m}$ by $60 \mu \mathrm{m}$ presented in Figure 2. The transmission area is the strip on the left while the receiver area is the strip on the right and the separation between the transmission zone and the receiver zone is $40 \mu \mathrm{m}$. In our simulations we always use the corresponding optimized grid loading area for each vesicle size as shown in Figure 4. Based on experimental results we assume the length of the microtubules is $10 \mu \mathrm{m}$ and that the number of vesicles a microtubule can load is half its length divided by the diameter of the vesicles. These parameters are summarized in Table 1.

Table 1: Simulation parameters for vesicular encapsulation

\begin{tabular}{ccc}
\hline \hline Vesicle's Diameter & Number of Squares in Grid & Microtubule's Maximum Load \\
\hline $250 \mathrm{~nm}$ & 1600 & 20 \\
$500 \mathrm{~nm}$ & 400 & 10 \\
$1 \mu \mathrm{m}$ & 100 & 5 \\
$2 \mu \mathrm{m}$ & 50 & 2 \\
\hline
\end{tabular}


Since there are lots of different parameters effecting the encapsulation process, we present three distinct set of results in the next four subsections. First, we consider the amount of liquid volume transported by each vesicle size (i.e. the liquid volume encapsulated inside vesicles). Since the molecular communication environment is aqueous, if we assume the concentration of the information particles is constant inside vesicles, the more liquid volume is transported the more information particles are transported. Second, we keep the size of the vesicles constant and study the effect of different concentrations of information particles on the number of transported information particles. Third, we consider a limited amount of lipid molecules available at the transmitter for generation of lipid vesicles, and study whether it is better to generate small number of large vesicles or numerous small vesicles.

\subsubsection{Effects of Vesicle Size}

Table 2: Number of vesicles to keep the liquid volume constant at $4.19 \mathrm{fL}$

\begin{tabular}{cc}
\hline \hline Vesicle's Diameter & Number of Vesicles \\
\hline $250 \mathrm{~nm}$ & 512 \\
$500 \mathrm{~nm}$ & 64 \\
$1 \mu \mathrm{m}$ & 8 \\
$2 \mu \mathrm{m}$ & 1 \\
\hline
\end{tabular}

We consider the effects of vesicle size on the amount of encapsulated liquid transported from the source to the destination. We assume the concentration of information particles in the liquid encapsulated by vesicles is constant. Since the number of information particles inside each vesicle is related to its concentration in the encapsulated liquid, the more liquid vol- 
ume is transported the more information particle is transported, assuming the concentration is constant.

Let $D$ be the inner diameter of the lipid vesicle. The volumetric capacity of the vesicle is therefore given by,

$$
V=\frac{4}{3} \pi\left(\frac{D}{2}\right)^{3}=\frac{1}{6} \pi D^{3} .
$$

For example, if we assume the diameter of the vesicle is $2 \mu \mathrm{m}$, the volume of the liquid inside a single vesicle is 4.19fL. If the vesicle's diameter is decreased to $1 \mu \mathrm{m}$, the liquid volume per single vesicle is $0.52 \mathrm{fL}$. Therefore, liquid volume inside a single $2 \mu \mathrm{m}$ vesicle is equivalent to liquid volume inside eight $1 \mu \mathrm{m}$ vesicles. In our simulations, to keep the total liquid volume at the transmitter constant at 4.19fL, different total number of vesicles is used for each vesicle size as shown in Table 2.

Figure 5 shows the average transported liquid volume with the standard deviation bars for time per channel use of 250 seconds. As the vesicle size increases, the average liquid volume transported increases. However, the standard deviation of the transported volume also increases as the liquid volume per single vesicle is greater. When the vesicle size is increased from the $250 \mathrm{~nm}$ to $1 \mu \mathrm{m}$ the average liquid volume transported increases consistently. However, the difference between the $1 \mu \mathrm{m}$ and $2 \mu \mathrm{m}$ vesicles is negligible. Moreover, the standard deviation of the $1 \mu \mathrm{m}$ vesicle is smaller. From these results we confirm that there exists an optimal vesicle size that can be found using our simulations. In this particular system the optimal vesicle size is $1 \mu \mathrm{m}$. 


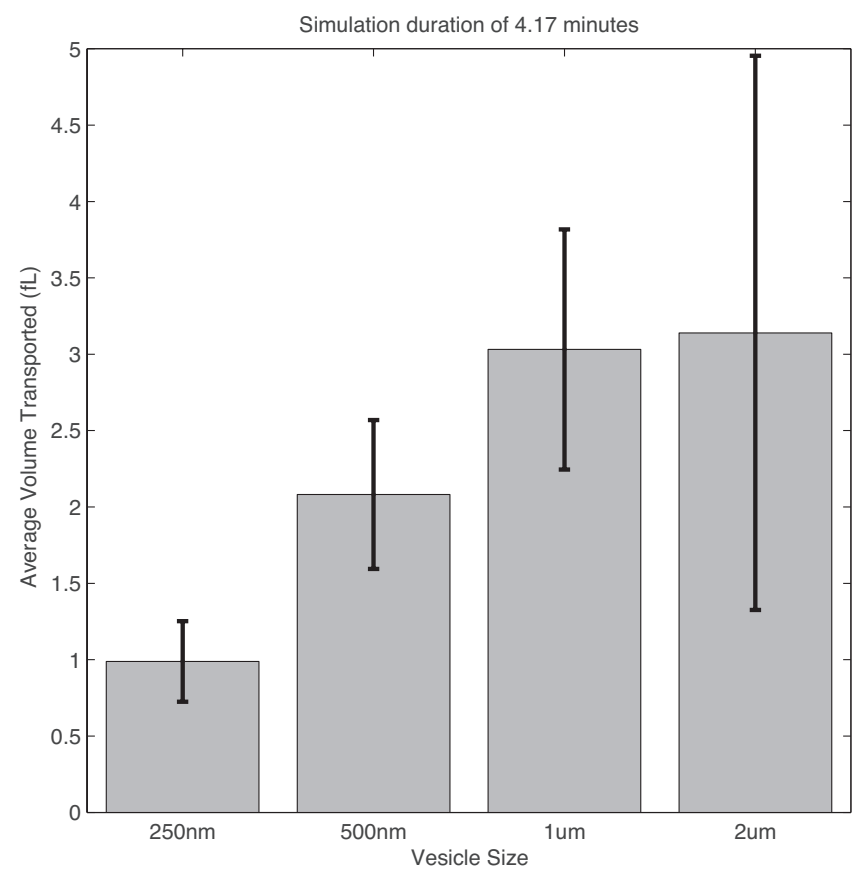

Figure 5: Average liquid volume transported for each vesicle size. The total liquid volume transmitted by the source is $4.19 \mathrm{fL}$. The lines show the standard deviation of the received volume. ( $\mathrm{T}=250 \mathrm{~s})$.

\subsubsection{Effects of Concentration of Information Particles}

We study the effects of the concentration of information particles inside vesicles in this section. We assume the vesicle size is constant at $1 \mu \mathrm{m}$, which is the optimal vesicle size based on the results in the previous section. We also assume that the total number of information particles transmitted by the source is constant. In particular we assume the transmitter releases 32 information particles. Let $\beta$ be the concentration of information particles inside vesicles. Then the number of information particles inside each vesicle 
Table 3: Number of vesicles and concentration of information particles ( $1 \mu \mathrm{m}$ vesicle)

\begin{tabular}{ccc}
\hline \hline Concentration (molecules per fL) & Info. Particles per Vesicle & Number of Vesicles \\
\hline 61.54 & 32 & 1 \\
30.77 & 16 & 2 \\
15.39 & 8 & 4 \\
7.69 & 4 & 8 \\
3.85 & 2 & 16 \\
1.92 & 1 & 32 \\
\hline
\end{tabular}

is given by

$$
z=\beta V
$$

where $z$ is the number of information particles per vesicle and $V$ is the volume of the vesicle.

For example, assuming the diameter of the vesicle is $1 \mu \mathrm{m}$ and concentration of $\beta=61.54$ molecules per $\mathrm{fL}$, the number of information particles per vesicle is $z=61.54 \times 0.52=32$. Therefore, if we assume the number of information molecules to be transmitted by the source is 32 , all the information particles can be encapsulated inside a single vesicle. Similarly, if the concentration is changed to $\beta=30.77$ molecules per fL, the number of information particles per vesicle is $z=30.77 \times 0.52=16$. Therefore, two vesicles are required to transmit 32 information particles. Following this approach, Table 3 summarizes different values used in our simulations.

Figure 6 shows the average number of information particles delivered with standard deviation bars based on each concentration for time durations per channel use of 250 seconds. One surprising outcome is that the average number of information particles delivered is very similar across different con- 


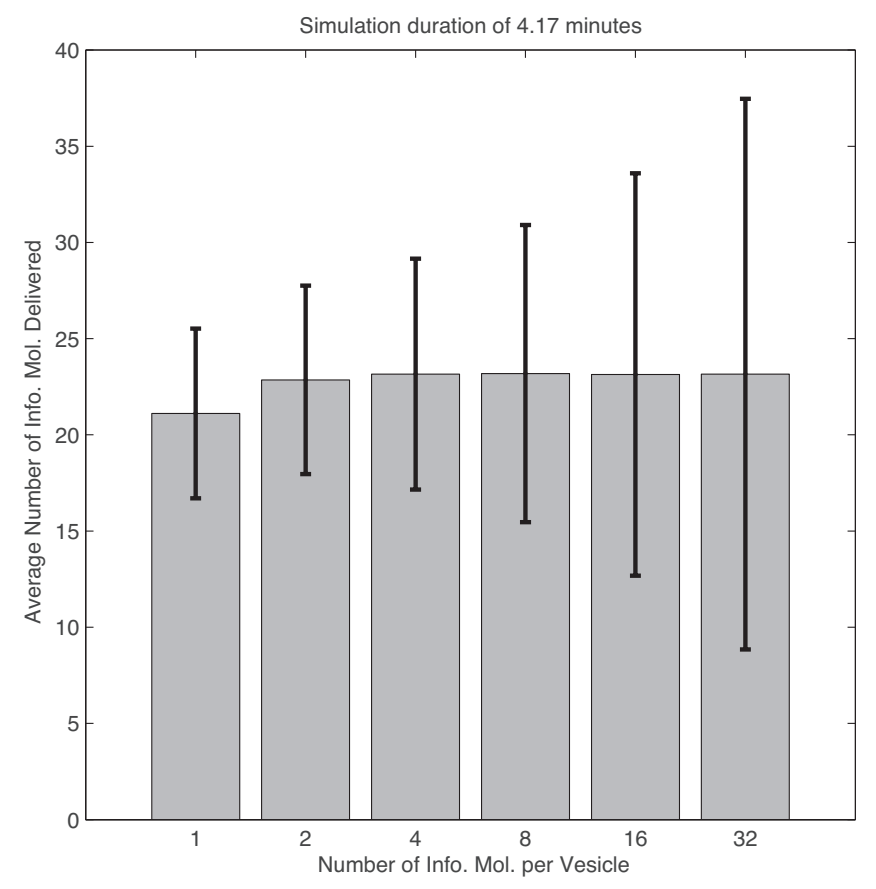

Figure 6: Average number of information molecules delivered for each concentration (i.e. number of information particles per vesicle). The total number of information particles transmitted is 32 . The lines show the standard deviation of the number of information particles received. $(\mathrm{T}=250 \mathrm{~s})$.

centration values. Moreover, as the concentration of information particles per vesicle is increased the standard deviation of the number of information particles delivered is increased.

\subsubsection{Effects of Number of Lipid Molecules}

In this section we study the effects of the number of lipid molecules on the number of information particles transported. The number of vesicles that can be generated based on the number of lipid molecules can be calculated using the equations presented in Section 3.2. By keeping the number of lipid 
Table 4: Number of vesicles that can be generated using $3.72 \times 10^{7}$ lipid molecules

\begin{tabular}{ccc}
\hline \hline Vesicle Size & Information Particles per Vesicle & Number of Vesicles \\
\hline $250 \mathrm{~nm}$ & 1 & 64 \\
$500 \mathrm{~nm}$ & 4 & 16 \\
$1 \mu \mathrm{m}$ & 16 & 4 \\
$2 \mu \mathrm{m}$ & 64 & 1 \\
\hline
\end{tabular}

molecules constant different number of vesicles can be generated for different vesicle sizes. For example, if the number of lipid molecules is about $3.72 \times 10^{7}$, applying Equation (14), a single $2 \mu \mathrm{m}$ vesicle can be generated. Similarly, using $3.72 \times 10^{7}$ lipid molecules, four $1 \mu \mathrm{m}$ vesicles can be generated. For the rest of this section we assume the number of lipid molecules is constant at $3.72 \times 10^{7}$ and the number of information particles to be transmitted is also constant at 64 . Table 4 shows the number of vesicles that can be generated as well as the number of information particles per vesicle.

Figure 7 shows the average number of information molecules delivered with standard deviation bars for time per channel use of 250 seconds. From the figure we can see that as the size of the vesicle is increased from $250 \mathrm{~nm}$ to $1 \mu \mathrm{m}$, the average number of information molecules delivered is increased. However, when the size of the vesicle is increased from $1 \mu \mathrm{m}$ to $2 \mu \mathrm{m}$, the average number of molecules delivered does not change significantly. The standard deviation of the number of information particles delivered increases as the size of the vesicle increases. From these results we conclude that there exist an optimal vesicle size for the channel considered. 


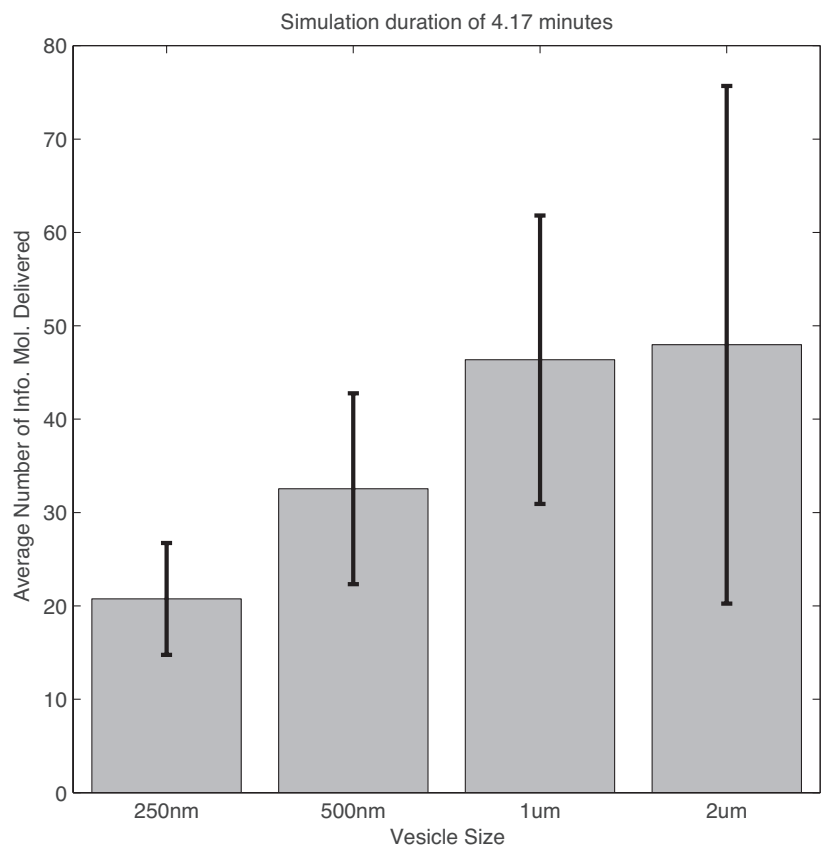

Figure 7: Average number of information molecules delivered for each vesicle size. The total number of information particles transmitted is 64 and the total number of lipid molecules is constant at $3.72 \times 10^{7}$. The lines show the standard deviation of the number of information particles received. ( $\mathrm{T}=250 \mathrm{~s})$.

\section{Information Theoretic Applications}

To show the flexibility of our transport model we use it in this section to estimate the channel capacity of active transport molecular communication system. We first describe channel capacity and achievable information rates. We then use both Monte Carlo simulations and the proposed transport model to estimate the channel capacity and compare the results. We also show that the optimizations performed in Section 3, increase the information rate and channel capacity. 


\subsection{Achievable Information Rates}

Previous work has considered molecular communication either as a timing channel problem (i.e., where information is encoded in the times when molecules are released) [6, 3]; as an inscribed matter problem (i.e., where information is encoded by transmitting custom-made particles, such as specific strands of DNA) [2]; or as a mass transfer problem (i.e., a message is transmitted by moving a number of particles from the transmitter to the receiver) $[3,4,5]$. In this paper, we consider information transmission as a mass transfer problem.

In the simplest possible conception of this scheme, the particles themselves are not information-bearing, and a message is conveyed in the number of particles released by the transmitter. For example, if a maximum of three particles may be used, from a traditional communication system perspective, we may form messages two bits long (i.e., $\left.\log _{2} 4\right)$ : "00" for 0 particle, "01" for 1 particle, "10" for 2 particles, and " 11 " for 3 particles. However, this message might not be perfectly conveyed to the receiver: given a time limit $T$ for the communication session, it is possible that some of the particles will not arrive at the receiver after $T$ has elapsed.

The maximum rate at which any communication system can reliably transmit information over a noisy channel is bounded by a limit called channel capacity [18]. In traditional communication systems, a source (transmitter) has a set of possible transmission symbols $\mathcal{X}$ and transmits a symbol $X \in \mathcal{X}$ per channel use, where, from the channel's perspective, $X$ is a random

variable given by probability distribution function $(\mathrm{PDF}) f_{X}(x)$. Because of the noise present in the channel, the destination (receiver) might not receive 


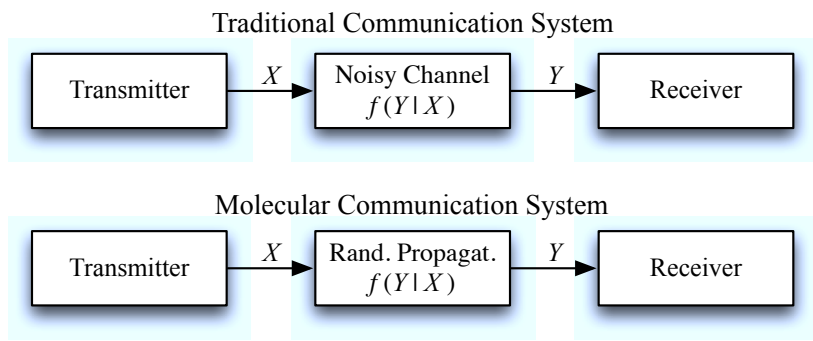

Figure 8: Traditional and molecular communication channels. In traditional communication the noisy channel limits the channel capacity, while in molecular communication the random propagation of information particles limits the channel capacity.

the symbol $X$ correctly. Let $\mathcal{Y}$ be the set of possible message symbols the destination can receive. Therefore, the destination receives a symbol $Y \in \mathcal{Y}$ per channel use, where $Y$ is a random variable given by $\operatorname{PDF} f_{Y}(y)$. This process is summarized in the top portion of Figure 8.

The channel capacity of such a communication channel can be calculated using mutual information $I(X ; Y)$, given by

$$
I(X ; Y)=E\left[\log _{2} \frac{f_{Y \mid X}(y \mid x)}{\sum_{x} f_{Y \mid X}(y \mid x) f_{X}(x)}\right],
$$

where, in this example, $f_{Y \mid X}(y \mid x)$ represents the probability of receiving symbol $y$ at the destination, given that symbol $x$ was transmitted by the source; $f_{X}(x)$ represents the probability of transmitting symbol $x$ at the source; and $E[\cdot]$ represents expectation. The channel capacity can be calculated using mutual information as,

$$
C=\max _{f_{X}(x)} I(X ; Y),
$$

where $C$ is the channel capacity, which is represented by the maximum value of mutual information over all possible transmission symbols' probability 
distributions.

The same concept can be applied to molecular communication systems, as shown in the bottom of Figure 8. Since we have assumed that messages are encoded in the number of particles, let $X \in \mathcal{X}$ represent the number of particles released into the medium by the transmitter (i.e. the symbol transmitted by the source), $Y \in \mathcal{Y}$ represent the number that arrive at the destination (i.e. the symbol received at the destination) once $T$ seconds have elapsed, and $x_{\max }$ be the maximum number of particles the transmitter can release per channel use. In other words, the set of possible transmission symbols and the set of possible received symbols are $\mathcal{X}=\mathcal{Y}=\left\{0,1,2, \cdots, x_{\max }\right\}$. Just like the traditional communication channel, from the channel's perspective, $X \in \mathcal{X}$ is a discrete random variable given by probability mass function $(\mathrm{PMF}) f_{X}(x), Y \in \mathcal{Y}$ is also a discrete random variable given by $\mathrm{PMF} f_{Y}(y)$, and a channel use is defined as $T$ second intervals between the transmission releases. Therefore in traditional communication system, the received symbols at the receiver are corrupted with noise from the environment, while in the molecular communication system, the received symbols are corrupted because of the random propagation of particles.

Clearly, there exists some PMF $f_{Y \mid X}(y \mid x)$ of the number of arrived particles given the number of transmitted particles. If this PMF is known, we can calculate mutual information for any $f_{X}(x)$. However, in order to calculate the channel capacity, we need to find the PMF $f_{X}(x)$ that maximizes mutual information. We can use the Blahut-Arimoto algorithm $[19,20]$ to find the PMF $f_{X}(x)$ such that, given $f_{Y \mid X}(y \mid x)$, mutual information is maximized. Therefore, if $\operatorname{PMF} f_{Y \mid X}(y \mid x)$ is known, we can calculate the channel capacity 
of the molecular communication system in a straightforward manner.

Finding the PMF $f_{Y \mid X}(y \mid x)$ is non-trivial because of the random motion of particles, as well as the shape and the geometry of the confined molecular communication channel. This PMF can be estimated using Monte Carlo simulations as proposed in $[8,5]$. Using this scheme, for a given number of particles released by the transmitter $(x)$, the motion of a microtubule is simulated for $T$ seconds and the number of particles delivered to the receiver $(y)$ is measured at the end of $T$ seconds. By repeating this process the PMF $f_{Y \mid X}(y \mid x)$ can be estimated for the time duration $T$ as shown in Algorithm 2. The whole simulation process can be repeated for different values of $x$. Since for each value of $X \in \mathcal{X}$ and $T$ a set of simulations is necessary, this process can be inefficient. However, employing our transport model this conditional probability can be calculated more efficiently. In the next two subsections, we use our transport model to estimate the channel capacity, and use Monte Carlo simulations to verify the optimizations proposed in Section 3.

\subsection{Analysis of the Transport Model}

In this section we compare our transport model to the Monte Carlo simulations proposed in [5] both in terms of simulation time and accuracy. We also show that the optimal loading area derived using our transport model does indeed increase the channel capacity of molecular communication channel.

We use the same molecular communication environment and simulation parameters used in Section 3.3. We assume the set of possible transmission symbols are $\mathcal{X}=\left\{0,1,2, \cdots, x_{\max }\right\}$, for some value of $x_{\max }$, where a transmission symbol $X \in \mathcal{X}$ is represented by release of $X$ information particles into the medium. All the released particles will be randomly distributed over 


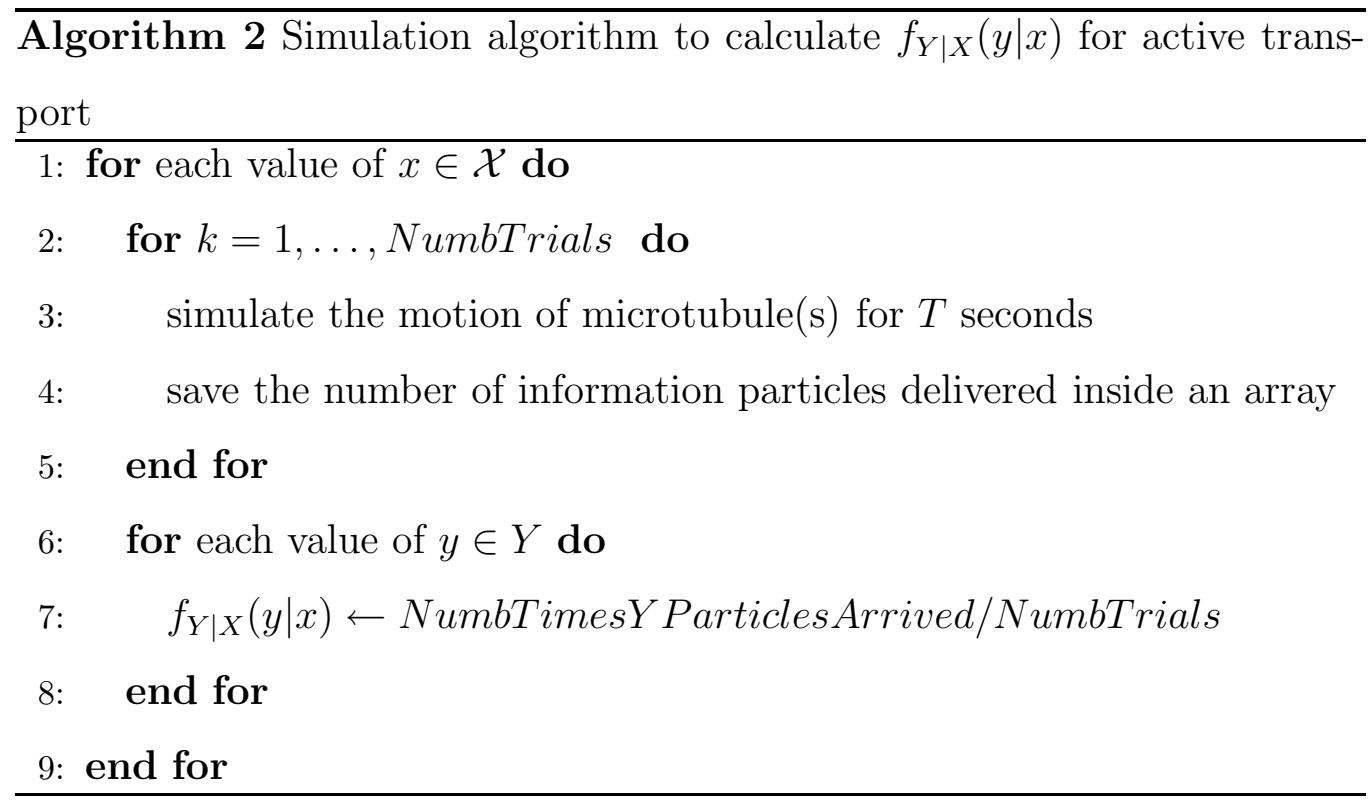

the transmission zone (i.e. grid transmission zone) and remain stationary until they are picked up by a microtubule for delivery. In case of Monte Carlo simulation we derive the $\operatorname{PMF} f_{Y \mid X}(y \mid x)$ by simulating the motion of the microtubules many times according to Algorithm 2, while we estimate the PMF $f_{Y \mid X}(y \mid x)$ using the proposed technique in case of transport model. We then use Blahut-Arimoto algorithm $[19,20]$ to find the $\operatorname{PMF} f_{X}(x)$, that would maximize the mutual information, and hence calculate the channel capacity based on each technique.

We assume the diameter of the information particles is $1 \mu \mathrm{m}$ and there are 100 squares in the grid loading area (i.e. there could be as much as 100 information particles in the loading strip). In case of Monte Carlo simulations, we assume the average length of the microtubules is $10 \mu \mathrm{m}$, and each microtubule can load up to 5 information particles in one trip from the transmission zone to the receiver zone. Notice that our transport model is not capable of cap- 


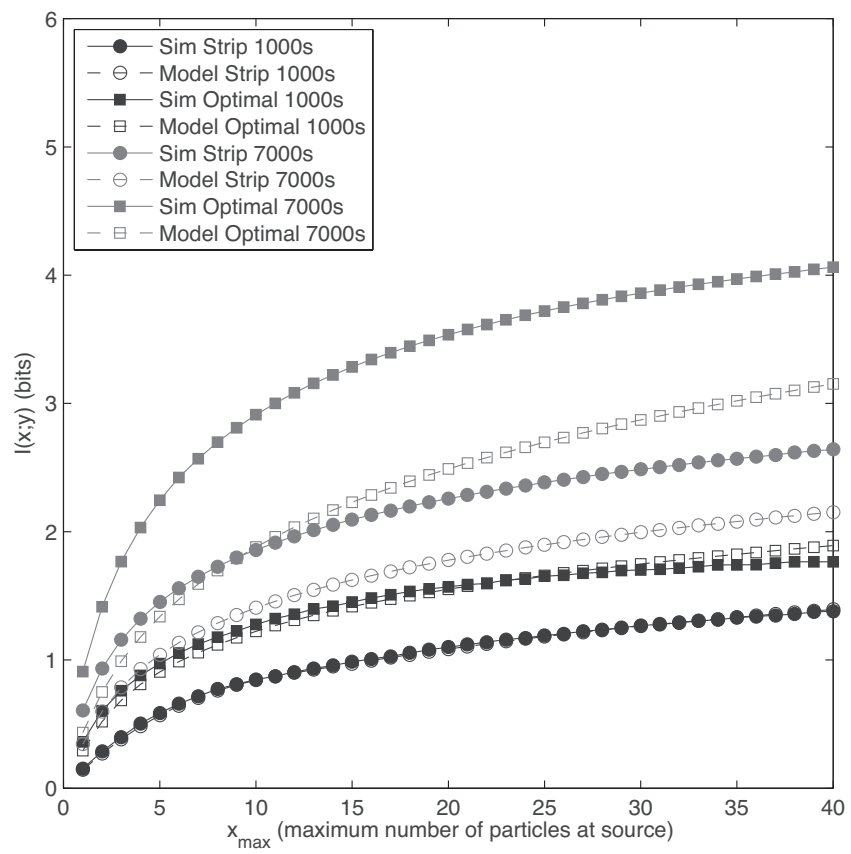

Figure 9: Mutual information (i.e. information rate) plot. Strip transmission zone plots are presented as circles and optimal transmission zone plots are presented as squares. The solid markers indicate simulated information rate and the empty markers present the information rate calculated using our model. The darker plots present the communication time duration of $1000 \mathrm{~s}$ and the lighter plots represent a time duration of $7000 \mathrm{~s}$. Note the very high accuracy of the model at $1000 \mathrm{~s}$.

turing this effect. However, as we show in our results the transport model is fairly accurate. These parameters are all selected based on experimental observations of ssDNA covered microtubules moving over a kinesin covered substrate. We also compare the information rate of the optimal loading area, generated using our transport model, to the strip loading area used in [5]. Recall that this optimal loading area is represented by the white squares in the bottom of the Figure 3. 
Figure 9 shows the mutual information (i.e. maximum achievable information rate) of two different transmission zones: strip transmission zone, shown using circles, and the optimal transmission zone, shown using squares. The mutual information is plotted against the $x_{\max }$ which represents the maximum number of possible transmission symbols. The mutual information is calculated by using both simulation (solid markers) and the transport model (empty markers). As we can see for small time durations, the transport model estimates the simulated mutual information closely. However, as time duration increases, the difference between the mutual information obtained through simulations and the transport model increases. This error is a byproduct of the independence assumptions made during the derivation of our transport model. Finally, we can see that the optimal transmission zone achieves a much higher information rate compared to the strip transmission zone as proposed by our analysis of our transport model.

\begin{tabular}{cc}
\multicolumn{2}{c}{ Table 5: Number of simulations required to estimate $f_{Y \mid X}(y \mid x)$} \\
\hline \hline Method of PMF Estimation & Number of Computer Simulations \\
\hline Monte Carlo Simulation & $2 \times 2 \times 40=160$ \\
transport model & 3 \\
\hline
\end{tabular}

We also compare the number of computer simulations required to estimate PMF $f_{Y \mid X}(y \mid x)$, for the plots in Figure 9, using both Monte Carlo simulations and the proposed transport model. The results are summarized in Table 5. Since there are 40 values of $x_{\max }$ and two time durations of $1000 \mathrm{~s}$ and $7000 \mathrm{~s}$, when Monte Carlo simulation is used to estimate the $\operatorname{PMF} f_{Y \mid X}(y \mid x)$, there are 80 sets of simulations required to generate the plot for the strip loading area and another 80 sets of simulations to generate the results for optimal 
loading area. However, using our transport model, two sets of simulations are necessary to calculate the PMF for $K$, the number of microtubule trips from the transmission zone to the receiver zone in times 1000s and 7000s, and one set of simulations to calculate the PMF for $V_{i}$, the probability that the $i$ th grid square is visited in one trip. These simulations can be used for both strip and optimal loading area and as the result no extra simulations are necessary.

When Monte Carlo simulation is employed, as the number of information particles released by the transmitter increases, the actual simulation times increases. For example, simulating one particle at the source take much less time than simulating 40 particles at the source. In this case, when one particle is assumed to be released, the simulation runs until time duration $T$ is simulated or until the single particle is delivered, while it must run until time duration $T$ is simulated or 40 particle are delivered, when 40 particles are assumed to be released. However, using the proposed transport model, the simulation times are constant regardless of the number of particles released by the transmitter. Therefore, using the proposed transport model the channel capacity can be calculated much quicker than the Monte Carlo simulations proposed in $[3,5]$. For example, using simulations written in Matlab [21], and average desktop computers equipped with Intel Core 2 Duo processors with different speeds ranging from $2.5 \mathrm{GHz}$ to $3.0 \mathrm{GHz}$, we used 40 different CPU cores to generate the Monte Carlo simulation results shown in Figure 9, for both the strip and optimal loading area, in about two weeks (almost one week for each transmission zone). However, the plots based on the transport model were generated using three CPU cores in about a day 
for both the strip and the optimal loading area.

\subsection{Effects of Vesicular Encapsulation}

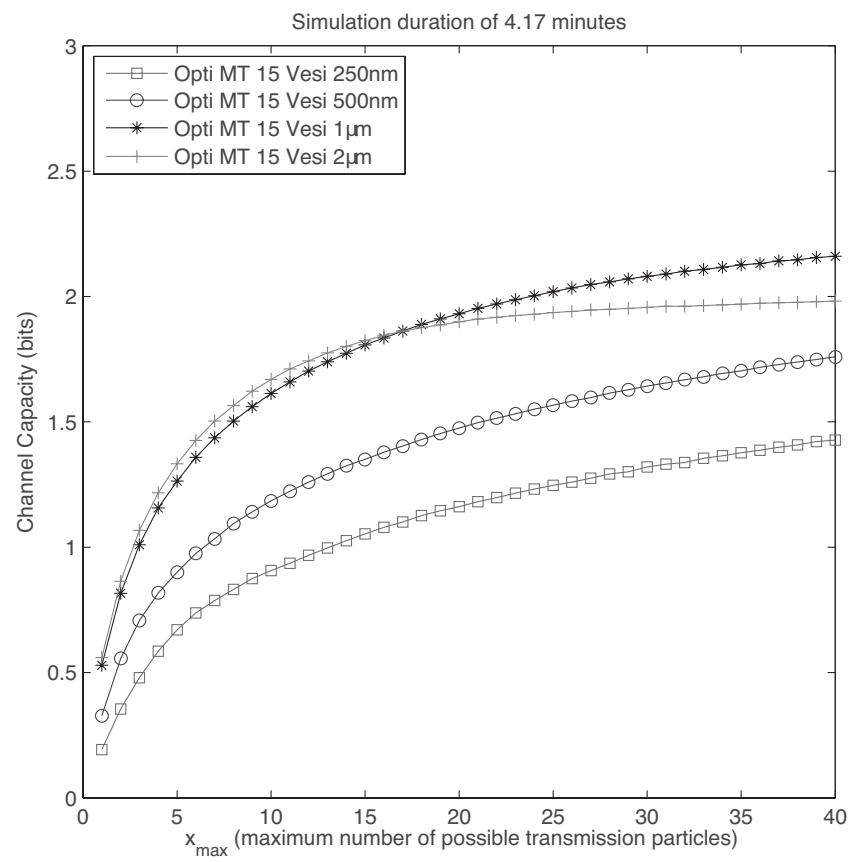

Figure 10: Channel capacity in bits for a single information particle encapsulated inside each lipid vesicle $(\mathrm{T}=250 \mathrm{~s})$.

In this section we consider the effects of vesicular encapsulation on achievable information rates. We use the same four vesicle sizes used in Section 3.3, as well as the same simulation environment and parameters. We assume only a single particle is encapsulated inside the lipid vesicles regardless of the vesicle's diameter. We assume the set of possible transmission symbols are $\mathcal{X}=\left\{0,1,2, \cdots, x_{\max }\right\}$, for some value of $x_{\max }$, where a transmission symbol $X \in \mathcal{X}$ is represented by release of $X$ information particles into the medium. 


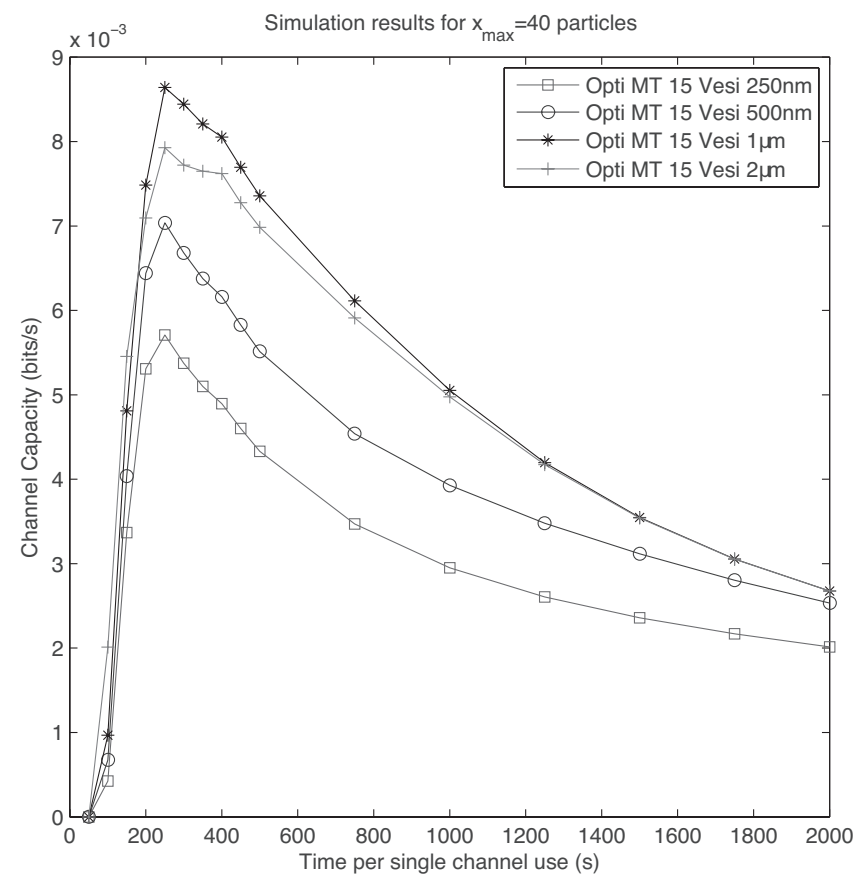

Figure 11: Channel capacity in bits per second versus time per channel use for a single information particle encapsulated inside each lipid vesicle.

All the released particles will be randomly distributed over the transmission zone (i.e. grid transmission zone) and remain stationary until they are picked up by a microtubule for delivery. To make the comparison we rely on Monte Carlo simulations.

The results are shown in Figure 10 for time duration per channel use of 250 seconds. From the graph we can see that as the size of the vesicles increase from $250 \mathrm{~nm}$ to $1 \mu \mathrm{m}$ the channel capacity of the microchannel increases. However, as the size increases from $1 \mu \mathrm{m}$ to $2 \mu \mathrm{m}$ the channel capacity does not increase. The effect is due to the difference in the shape of the transmission zone as shown in Figure 4. Because $2 \mu \mathrm{m}$ squares cover a larger area, 
they spread across the whole transmission area including the center parts where the probability of a microtubule visit is every low as shown in Figure 3.

In Figure 11 the channel capacity in bits per second is plotted against different time durations per channel use for $x_{\max }=40$. From the figure again we can see that as the size of the vesicle is increased from $250 \mathrm{~nm}$ to $1 \mu \mathrm{m}$ the curve's peak is increased. However, $1 \mu \mathrm{m}$ vesicles achieve a higher peak than the $2 \mu \mathrm{m}$ vesicles and hence have a higher channel capacity in bits per second. This is again because of the shape of the transmission area which in the case of $2 \mu \mathrm{m}$ vesicle is not optimized.

Based on the results we conclude that encapsulation of information vesicles using lipid vesicles improves the channel capacity. This effect is due to the small size of the information particles which are typically molecules. For example if no encapsulation is employed the size of a typical information particle would be $10 \mathrm{~nm}$. Therefore, based on the presented results we can see that $10 \mathrm{~nm}$ squares in the grid loading area would have a much lower channel capacity compared to the larger squares of vesicular encapsulation. However, depending on the shape and the dimensions of the transmission area, there is an optimal vesicle size. This effect is particularly evident in our simulations as we increase the vesicle size from $1 \mu \mathrm{m}$ to $2 \mu \mathrm{m}$. Therefore, we conclude that the optimal vesicle size for this particular channel is $1 \mu \mathrm{m}$, which is in accordance with our results in Section 3.3. 


\section{Conclusion}

In this work we presented a simple transport model for molecular communication system employing active transport propagation. We showed that our model could be used to study and optimize molecular communication systems. As an example, we optimized the shape of the transmission area, and considered vesicular encapsulation. We showed that for a given communication channel there is an optimal vesicle size. As an application, we used our transport model to estimate the channel capacity of a molecular communication channel. We considered a molecular communication system, in a rectangular confined space, employing active transport as proposed in [9]. In order to estimate the channel capacity of such a communication system, previous works $[3,5]$, had relied on Monte Carlo simulations of the motion of microtubule filaments. These simulations can be time consuming since for each set of possible transmission symbols as well as channel time durations, a different set of simulations is necessary. However, by employing our transport model, we showed that the channel capacity could be estimated in a computationally efficient manner. The capacity estimation obtained using the proposed transport model is very close to the Monte Carlo simulation estimated channel capacity for small channel time durations. However, as channel time duration increases, the estimation error increases for the proposed transport model. We also showed that by optimizing the shape of the transmission zone using our transport model, channel capacity increases. Moreover, we showed that using an optimal vesicle size, derived using the transport model, increases the channel capacity. 


\section{References}

[1] S. Hiyama, Y. Moritani, Molecular communication: harnessing biochemical materials to engineer biomimetic communication systems, Nano Communication Networks 1 (1) (2010) 20-30.

$[2]$ L. C. Cobo, I. F. Akyildiz, Bacteria-based communication in nanonetworks, Nano Communication Networks 1 (4) (2010) 244-256.

[3] A. W. Eckford, Timing information rates for active transport molecular communication, in: Nano-Net, Vol. 20 of Lecture Notes of the Institute for Computer Sciences, Social Informatics and Telecommunications Engineering, Springer Berlin Heidelberg, 2009, pp. 24-28.

[4] M. U. Mahfuz, D. Makrakis, H. T. Mouftah, On the characterization of binary concentration-encoded molecular communication in nanonetworks, Nano Communication Networks 1 (4) (2010) 289-300.

[5] N. Farsad, A. W. Eckford, S. Hiyama, Y. Moritani, Information rates of active propagation in microchannel molecular communication, in: Proc. of the 5th International ICST Conference on Bio-Inspired Models of Network, Information, and Computing Systems, Boston, MA, 2010, p. 7.

[6] A. W. Eckford, Nanoscale communication with brownian motion, in: Proc. of the 41st Annual Conference on Information Sciences and Systems, Baltimore, MD, 2007, pp. $160-165$.

[7] S. Matosevic, B. M. Paegel, Stepwise synthesis of giant unilamellar vesicles on a microfluidic assembly line, Journal of the American Chemical Society 133 (9) (2011) $2798-2800$.

[8] A. W. Eckford, N. Farsad, S. Hiyama, Y. Moritani, Microchannel molecular communication with nanoscale carriers: Brownian motion versus active transport, in: Proc. of the IEEE International Conference on Nanotechnology, Seoul, South Korea, 2010, p. 5 .

[9] S. Hiyama, R. Gojo, T. Shima, S. Takeuchi, K. Sutoh, Biomolecular-motor-based nano- or microscale particle translocations on dna microarrays, Nano Letters 9 (6) (2009) 2407-2413.

[10] S. Hiyama, Y. Moritani, R. Gojo, S. Takeuchi, K. Sutoh, Biomolecular-motor-based autonomous delivery of lipid vesicles as nano- or microscale reactors on a chip, Lab on a Chip 10 (20) (2010) 2741-2748.

[11] B. Atakan, O. Akan, An information theoretical approach for molecular communication, in: Proc. of the 2nd International Conference on Bio-Inspired Models of Network, Information and Computing Systems, Budapest, Hungary, 2007, pp. 33-40. 
[12] M. J. Moore, T. Suda, K. Oiwa, Molecular communication: modeling noise effects on information rate, IEEE Transactions on NanoBioscience 8 (2) (2009) 169-180.

[13] M. Pierobon, I. F. Akyildiz, A physical end-to-end model for molecular communication in nanonetworks, IEEE Journal on Selected Areas in Communications 28 (4) (2010) 602-611.

[14] T. Nitta, A. Tanahashi, M. Hirano, H. Hess, Simulating molecular shuttle movements: Towards computer-aided design of nanoscale transport systems, Lab on a Chip 6 (7) (2006) 881-885.

[15] T. Nitta, A. Tanahashi, M. Hirano, In silico design and testing of guiding tracks for molecular shuttles powered by kinesin motors, Lab on a Chip 10 (11) (2010) $1447-1453$.

[16] S. X. Chen, J. S. Liu, Statistical applications of the poisson-binomial and conditional bernoulli distributions, Statistica Sinica 7 (1997) 875-892.

[17] C. Huang, J. T. Mason, Geometric packing constraints in egg phosphatidylcholine vesicle, Proceedings of National Academy of Science USA 75 (1) (1978) 308-310.

[18] T. M. Cover, J. A. Thomas, Elements of Information Theory 2nd Edition, 2nd Edition, Wiley-Interscience, 2006.

[19] R. Blahut, Computation of channel capacity and rate-distortion functions, IEEE Transactions on Information Theory 18 (4) (1972) 460-473.

[20] S. Arimoto, An algorithm for computing the capacity of arbitrary discrete memoryless channels, IEEE Transactions on Information Theory 18 (1) (1972) 14-20.

[21] MATLAB, version 7.10.0 (R2010a), The MathWorks Inc., Natick, Massachusetts, 2010 . 